\title{
Bivariate Histogram Segmentation of Simultaneous Neutron and X-ray Tomography for Improved Compositional and Structural Determination of Source Rock Shales
}

Jacob LaManna ${ }^{1}$, Jin-Hong Chen ${ }^{2}$, Stacey Althaus ${ }^{2}$, Yun Liu ${ }^{1}$, Daniel Hussey ${ }^{1}$ and David Jacobson ${ }^{1}$

${ }^{1}$ National Institute of Standards and Technology, Gaithersburg, Maryland, United States, ${ }^{2}$ Aramco Services Company, Houston, Texas, United States

Shale gas has seen an incredible growth in the United States and worldwide due to improvements in horizontal drilling and hydraulic fracturing. Significant improvements to gas extraction from reservoirs can be made with improved understanding of the distribution and transport pathways for hydrocarbon flow in shales. Many techniques to understand the distribution of organic matter and minerals within shale, such as FIB-SEM and nano X-ray tomography, are at the pore scale and may not provide distributions representative on the core or well length scales. Neutron and X-ray tomography are complementary, nondestructive methods to probe the three-dimensional structure of shales at the core scale. Neutrons have a strong sensitivity to hydrogen and therefore organic matter while X-rays are sensitive to increasing atomic mass such as iron rich minerals. To fully leverage the complementarity of neutrons and X-rays, NIST has developed the Neutron and X-ray Tomography (NeXT) system for truly simultaneous multimodal tomography [1]. NeXT orients a $90 \mathrm{keV}$ microfocus X-ray generator perpendicular to the neutron beam. Software has been developed to account for the 90-degree offset of the reconstructed volumes and fully register the volumes for a 1 to 1 comparison between modalities.

Typical methods for segmentation typically revolve around 1D histogram segmentation, thresholding, or manual identification, i.e. painting. Thresholding or histogram segmentation can become difficult if the regions of interest lack enough contrast difference for proper identification and manual identification is incredibly labor intensive and time consuming. NIST has developed a bivariate histogram segmentation tool that leverages the contrast differences of the two modalities from NeXT to overcome the shortcomings of the typical segmentation methods. This tool loads in the registered neutron and X-ray reconstructed volumes and creates a 2D histogram of neutron versus X-ray greyscale values. The tool then allows the researcher to draw out regions of interest around peaks on the $2 \mathrm{D}$ histogram to tag those values as particular phases/materials. Upon completion of identification of all desired phases, the program will output one volume of fully colorized slice images and additionally, binary volumes for each identified phase. The typical active time required of a researcher using the tool is 15 to 30 minutes, which is a significant reduction in the required effort compared to some segmentation methods.

To demonstrate the output of the bivariate histogram segmentation tool, several shale plugs previously scanned with the NeXT system [2-4] were evaluated with the tool. Figure 1(a \& b) show corresponding neutron and X-ray reconstruction slices through the center of one of the plugs. The slices show the strong contrast differences between neutrons and X-rays. Brighter areas in the neutron slice (Figure 1a) are highly organic rich regions, with the brightest areas appearing nearly invisible in the X-ray due to the low atomic mass of organic matter. In contrast, brighter areas in X-ray are regions with higher atomic mass. Figure 1(c \& d) show the original 1D histogram segmentation efforts with each volume segmented independent of the other volume. Figure 2 gives the significantly improved segmentation results by combining the neutron and X-ray information into a single, combined segmentation. With the bivariate histogram segmentation tool it is possible to not only highlight the regions of high organic content and high- $\mathrm{Z}$ 
minerals, as seen in the earlier work, but it is now possible to subdivide further into regions of moderate organic content, organic deficient regions, moderately high-Z minerals, and the overall composite volume.
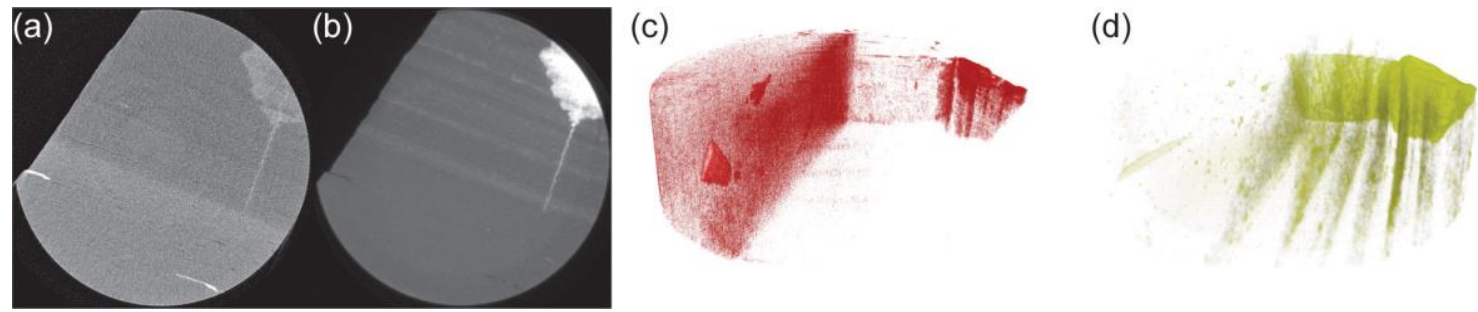

Figure 1. (a) slice through center of the neutron volume, brighter regions indicate higher organic content, (b) slice through center of the X-ray volume, brighter regions indicate higher atomic mass, (c \& d) original independent $1 \mathrm{D}$ histogram segmentation results showing regions with high organic content (red) and higher atomic mass (yellow).

(a)

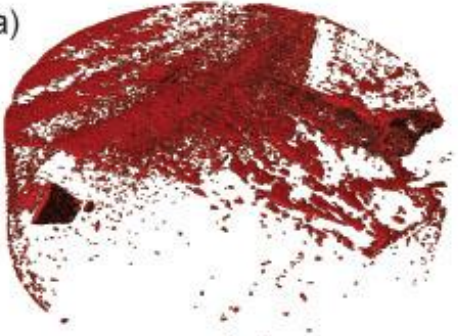

(d)

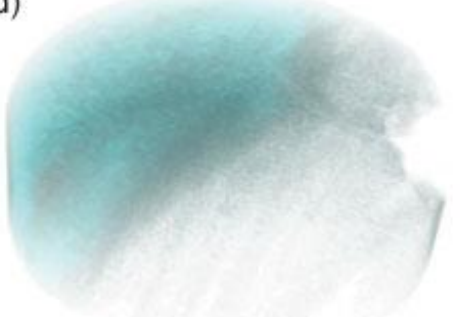

(b)

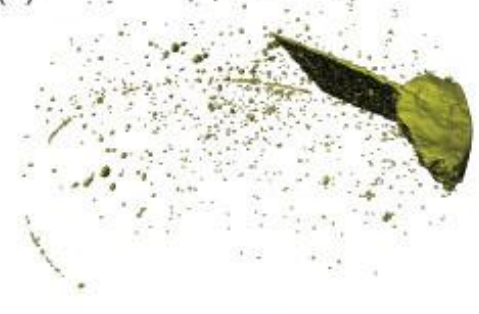

(e)

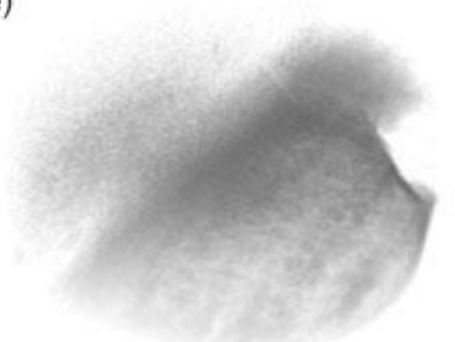

(c)

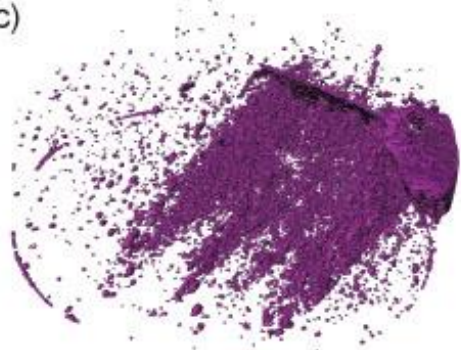

(f)

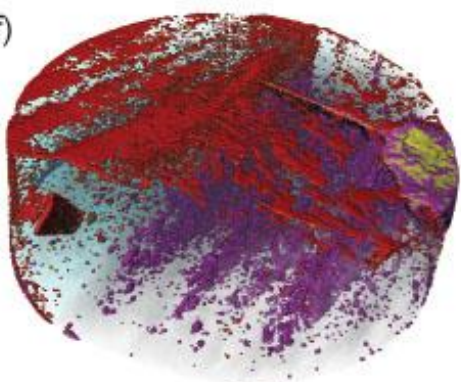

Figure 2. Results of the bivariate histogram segmentation tool: (a) Highly organic rich material, (b) pyrite, (c) moderate- $\mathrm{Z}$ material (most likely an aluminosilicate), (d) moderate organic content material (volume shown with increased transparency to make internal structure more visible), (e) low/no organic content material (volume shown with increased transparency to make internal structure more visible), (f) composite image of all regions.

\section{References}

[1] LaManna, J.M. et al., "Neutron and X-ray tomography (NeXT) system for simultaneous, dual modality tomography", Review of Scientific Instruments, 88(11), pp113702, 2017. [2] Chiang, W.S. et al., "Simultaneous neutron and X-ray imaging of 3D kerogen and fracture structures in shales", SPWLA $58^{\text {th }}$ Annual Logging Symposium, 2017. [3] Chiang, W.S. et al., "Simultaneous neutron and X-ray imaging of 3D structure of organic matter and fracture in shales", Petrophysics, 59(02), pp153-161, 2018. [4] Li, W. et al., "3D core-scale organic and mineral material characterization of source rocks with simultaneous neutron and X-ray imaging", Microscopy and Microanalysis, 23(S1), pp2174-2175, 2017. 